\title{
Endovascular therapy choices for different sites of delayed postoperative arterial hemorrhage after hepatobiliary pancreatic surgery: a retrospective study
}

\author{
Xiaohe Yu ${ }^{1,2 \#}$, Xue Liu ${ }^{1 \#}$, Jian Huang ${ }^{1}$, Shuqun Shen ${ }^{1}$, Naijian Ge ${ }^{1}$, Yefa Yang ${ }^{1}$, Hua Wang ${ }^{1,3}$ \\ ${ }^{1}$ Department of Radioactive Intervention, Eastern Hepatobiliary Surgery Hospital, Second Military Medical University, Shanghai, China; \\ ${ }^{2}$ Department of Critical Care Medicine, Qingpu Branch of Zhongshan Hospital Affiliated to Fudan University, Shanghai, China; ${ }^{3}$ Intervention and \\ Vascular Surgery, Jiading Branch of Shanghai First People's Hospital, Shanghai, China \\ Contributions: (I) Conception and design: X Yu, X Liu, H Wang; (II) Administrative support: N Ge, Y Yang; (III) Provision of study materials or \\ patients: X Yu, X Liu, Y Yang, H Wang; (IV) Collection and assembly of data: Jian Huang, Shuqun Shen; (V) Data analysis and interpretation: X Yu, \\ X Liu, Y Yang; (VI) Manuscript writing: All authors; (VII) Final approval of manuscript: All authors. \\ \#These authors contributed equally to this work. \\ Correspondence to: Hua Wang; Yefa Yang. Department of Radioactive Intervention, Eastern Hepatobiliary Surgery Hospital, Second Military Medical \\ University, 225 Changhai Road, Shanghai 200438, China. Email: whzhfq@hotmail.com; yangyefa66@163.com.
}

Background: Angiography and subsequent endovascular therapy is an effective technique for delayed postoperative arterial hemorrhage (PAH) after hepatobiliary pancreatic surgery. In this research, we aimed to evaluate endovascular therapy choices for different sites of delayed PAH after hepatobiliary pancreatic surgery.

Methods: A total of 85 patients with delayed PAH who underwent endovascular therapy at the Department of Radioactive Intervention of Eastern Hepatobiliary Surgery Hospital were retrospectively enrolled. According to the hemorrhage site, participants were divided into 3 groups, all of whom then received embolization, covered stent placement, or a combination of both. Ongoing or recurrent hemorrhages, intervention times, complications associated with intervention, and mortality rate were documented. The chi-squared $\left(\chi^{2}\right)$ test was used for statistical analysis.

Results: A total of 22 participants with arterial branch hemorrhage underwent superselective embolization. Overall, 81.8\% (18/22) of patients underwent embolization once. The successful hemostasis rate was $77.3 \%$ $(17 / 22)$, and the mortality rate was $13.6 \%$ (3/22). A total of 53 participants with arterial trunk hemorrhage underwent embolization or covered stent placement. The rate of multi-time intervention, failure to achieve hemostasis, complications associated with intervention, and mortality was lower in the stent group than in the embolization group, and there was a significant difference in complications between the 2 groups $\left(\chi^{2}=4.93\right.$, $\mathrm{P}=0.026$ ). Among a total of 10 patients with multisite hemorrhage who underwent embolization, covered stent placement, or a combination, the successful hemostasis rate was $20 \%$; and the mortality rate was $70 \%$.

Conclusions: Superselective embolization is a safe treatment method for arterial branch hemorrhage, and covered stent placement may be a better choice for arterial trunk hemorrhage. Verification of these findings is required via additional large population studies.

Keywords: Endovascular therapy; delayed postoperative arterial hemorrhage; hepatobiliary pancreatic surgery; covered stent placement; embolization

Submitted Jul 16, 2021. Accepted for publication Sep 15, 2021.

doi: $10.21037 /$ gs-21-521

View this article at: https://dx.doi.org/10.21037/gs-21-521 


\section{Introduction}

Postoperative arterial hemorrhage (PAH) is an uncommon but potentially life-threatening complication following hepatobiliary pancreatic surgery (1). Based on the occurrence time, $\mathrm{PAH}$ after hepatobiliary pancreatic surgery can be classified as an early- and delayed-phase hemorrhage. In the early phase, hemorrhage is usually caused by technical failure or coagulopathy and requires repeat laparotomy and surgical hemostasis $(1,2)$. Delayed hemorrhage can result from vessel erosion in the case of pancreatic leakage, intraabdominal infection with vessel involvement, or vascular injury during resection and pseudoaneurysm formation in the postoperative interval (3-6). Due to the diversity of bleeding sites and postoperative adhesions, it is reasonably difficult to precisely identify the hemorrhaging artery by surgical exploration (7). Recent studies have confirmed that immediate angiography and subsequent endovascular therapy is a safe and effective technique, and it should be used as the first-line diagnostic and treatment choice for delayed PAH after hepatobiliary pancreatic surgery $(2,8)$. However, standardized endovascular treatments for its management do not exist. Transarterial embolization (TAE) and covered stent placement are 2 mainstream treatments for delayed PAH (9-11), but how to discriminatively choose between the 2 methods according to different sites of delayed PAH is still unclear (8). This study aimed to evaluate different endovascular therapy choices for different sites of delayed PAH after hepatobiliary pancreatic surgery. We present the following article in accordance with the STROBE reporting checklist (available at https://dx.doi. org/10.21037/gs-21-521).

\section{Methods}

\section{Participants}

This study retrospectively analyzed 88 consecutive patients after hepatobiliary pancreatic surgery who experienced delayed PAH as demonstrated by digital subtraction angiography (DSA) at the Department of Radioactive Intervention of Eastern Hepatobiliary Surgery Hospital, Second Military Medical University in Shanghai, China, between July 2016 and June 2019. Surgical hemostasis was conducted on 2 participants, because the hemorrhage site was located in the stem of the superior mesenteric artery (SMA), which meant that TAE or covered stent placement would lead to bowel necrosis. A single participant had a hepatic artery
Table 1 Clinical characteristics of the 85 participants with delayed PAH after hepatobiliary pancreatic surgery who underwent endovascular therapy

\begin{tabular}{lc}
\hline Clinical characteristics & Value \\
\hline Male/female & $57 / 28$ \\
Age, year & $60[37-77]$ \\
Diagnosis & 35 \\
Cholangiocarcinoma & 15 \\
Benign biliary tract disease & 13 \\
Duodenal ampullary tumor & 11 \\
Pancreatic disease & 7 \\
Hepatic tumor & 4 \\
Gallbladder disease & $15 \pm 11.0$ \\
Type of surgery & 39 \\
Pancreatic surgery & 30 \\
Biliary tract surgery & 7 \\
Liver resection & 34 \\
Biliary tract exploration & 4 \\
Distal pancreatectomy & 3 \\
Radical cholecystectomy & \\
Other complications of surgery & \\
Yes & \\
No & 3 \\
\hline Bleeding time after surgery, day & \\
\hline
\end{tabular}

$\mathrm{PAH}$, postoperative arterial hemorrhage.

pseudoaneurysm after liver transplantation, for which covered stent placement failed and a second surgery was performed. The other 85 participants were initially treated by endovascular therapy, and their detailed clinical characteristics are shown in Table 1. According to bleeding site, the 85 participants who were treated by endovascular therapy were divided into 3 groups: arterial branch hemorrhage, arterial trunk hemorrhage, and multisite hemorrhage (Figure 1). Multisite hemorrhage was defined as more than 1 bleeding site identified in the first DSA or a new bleeding site upon repeated DSA. All procedures performed in this study involving human participants were in accordance with the Declaration of Helsinki (as revised in 2013). The study was approved by ethics board of Eastern Hepatobiliary Surgery Hospital, and informed consent was taken from all the patients. 


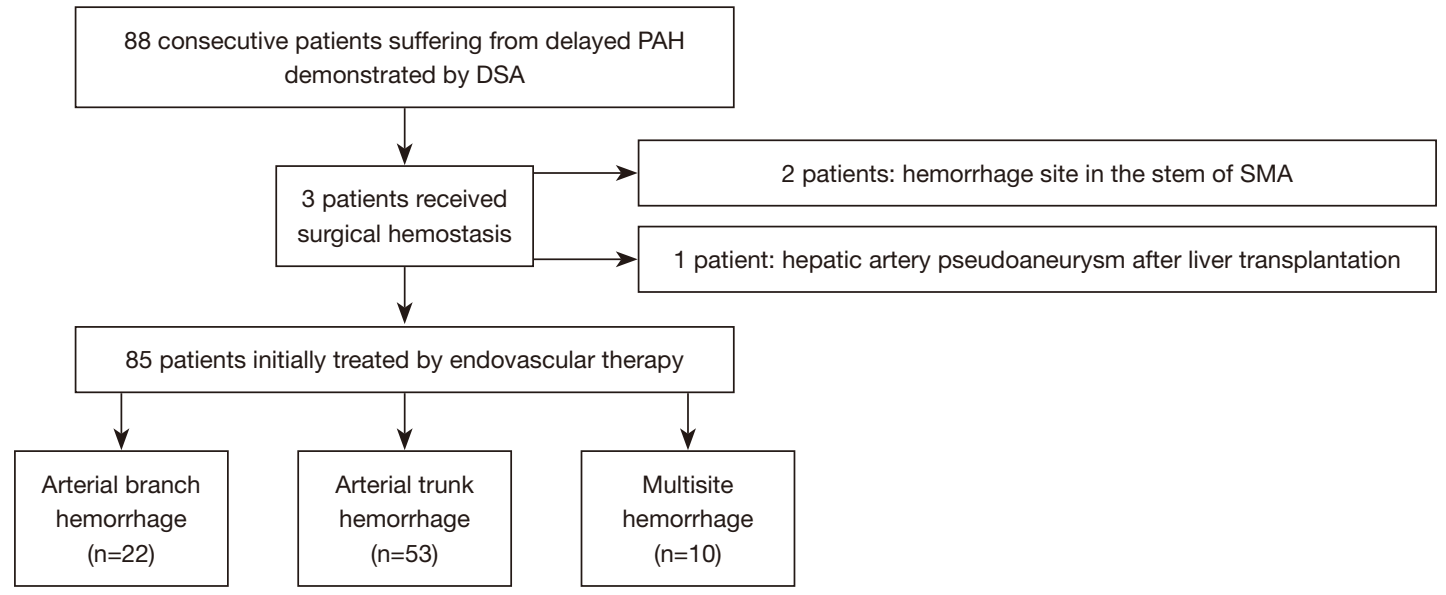

Figure 1 A flow diagrams showed the initial number of participants and those excluded for any reason, then how to divide into groups.

\section{Diagnostic angiography}

The angiography was performed using a GE Innova IGS 540 system (General Electric Company, Boston, MA, USA). For vascular access, a 5-F sheath (Terumo Corp., Tokyo, Japan) was inserted into the right femoral artery. Then, selective and superselective angiograms were performed, including the late portal venous phase. A selective angiography was performed using a 5-F Yashiro or RH catheter (Terumo Corp., Japan) positioned in the celiac trunk and superior mesenteric artery with an automated injection of $30 \mathrm{~mL}$ of iodinated contrast material at a rate of $5 \mathrm{~mL} / \mathrm{s}$ with 300 psi of pressure. A superselective angiography was performed at a rate of $2.5 \mathrm{~mL}$ using a 2.8-F microcatheter system (Renegade HiFlo Fathom ${ }^{\mathrm{TM}}$, Boston Scientific, Natick, MA, USA) with $500 \mathrm{psi}$ of pressure. The signs of delayed PAH included local abnormal vessels, extravasation of the contrast medium, or pseudoaneurysm. The angiography was reviewed by 2 experienced interventional radiologists, and decisions were made based on consensus.

\section{Superselective embolization}

Embolization was performed with a superselective catheter (Renegade Hi-Flo Fathom ${ }^{\mathrm{TM}}$, Boston Scientific, USA) position by coil embolization (Tornado Embolization Microcoil $^{\mathrm{TM}}$, Cook Medical, Bloomington, IN, USA or Interlocking Detachable Coils, Boston Scientific, Cork, Ireland), microspheres (500-700 or 700-900 $\mu \mathrm{m}$; Embosphere, Merit Medical Systems, South Jordan, UT, USA), or a gelatin sponge particle embolic agent (Gel
Foam, Alicon Pharm., Hangzhou, China). The coils were placed until a complete exclusion of the bleeding site was obtained, which was defined by an absence of the vascular abnormality on a repeat angiography. Microspheres or a gelatin sponge embolization was most often used for coilassisted embolization. Hemorrhages that originated from the branches of SMA or pancreatic artery were occluded by coil only, due to the risk of microspheres or a gelatin sponge embolization leading to bowel or pancreatic necrosis.

\section{Covered stent placement}

A covered stent was placed for arterial trunk hemorrhage to exclude the vessel leakage or pseudoaneurysm and maintain distal organ perfusion. After the guidewire crossed the vessel leakage or pseudoaneurysm to the distal artery, a Super Stiff guidewire (Boston Scientific, Marlborough, MA, USA) was used to exchange the former guidewire. Then, an 8-F or 9-F sheath (Terumo, Japan) was placed for the delivery of stent grafts. A vascular stent graft (Fluency Plus, C.R. Bard Company, Franklin Lakes, NJ, USA) of 6 or $8 \mathrm{~mm}$ in diameter and $40-80 \mathrm{~mm}$ in length was used. After stent placement, a repeat angiography was applied to verify the complete exclusion of the vessel leakage or pseudoaneurysm.

\section{Follow up and statistical analysis}

Complications associated with hepatobiliary pancreatic surgery were documented. Ongoing or recurrent 
Table 2 Distribution of different sites of delayed PAH after hepatobiliary pancreatic surgery

\begin{tabular}{lc}
\hline Site of hemorrhage & Number \\
\hline Arterial branch hemorrhage & 22 \\
Branch of hepatic artery & 9 \\
Branch of superior mesenteric artery & 7 \\
Branch of left gastric artery & 4 \\
Branch of dorsal pancreatic artery & 2 \\
Arterial trunk hemorrhage & 53 \\
Stump of gastroduodenal artery & 27 \\
Common hepatic artery & 12 \\
Proper hepatic artery & 6 \\
Right hepatic artery & 5 \\
Aberrant right hepatic artery & 2 \\
Splenic artery & 1 \\
Multi-site hemorrhage & 10 \\
\hline
\end{tabular}

$\mathrm{PAH}$, postoperative arterial hemorrhage.

Table 3 Outcome of super selective embolization in artery branch hemorrhage

\begin{tabular}{lc}
\hline Outcome & $\mathrm{n}=22$ \\
\hline Times of intervention & 18 \\
Single & 4 \\
Multiple & \\
Hemostasis & 5 \\
No & 17 \\
Yes & \\
Complications of intervention & 21 \\
No & 1 \\
Yes & \\
Survival & 3 \\
No & 19 \\
Yes & \\
\hline
\end{tabular}

hemorrhages; interventions times; treatment following rebleeding; complications associated with intervention therapy (acute liver failure, bowel necrosis, and pancreatic necrosis); and mortality that occurred during the study were documented. The mean follow-up time was 97 days
(21-189 days). The software SPSS 19.0 (IBM Corp., Armonk, NY, USA) was used for all statistical analyses. Differences among categorical variables were analyzed for statistical significance using a chi-squared $\left(\chi^{2}\right)$ analysis. All $\mathrm{P}$ values were 2 -sided, and $\mathrm{P}<0.05$ was the threshold for statistical significance.

\section{Results}

A total of 88 participants manifested delayed PAH after hepatobiliary pancreatic surgery was documented. Among them, 2 participants underwent surgical hemostasis, because the hemorrhage position was located in the stem of the SMA, and TAE or covered stent placement would have led to bowel necrosis. A single participant had a hepatic artery pseudoaneurysm after liver transplantation, for which covered stent placement failed and a second surgery was performed. Eventually, 85 participants initially treated by endovascular therapy after hemorrhage were recruited to this study. Details of the clinical characteristics of the 85 participants are shown in Table 1 .

There were 22 participants with arterial branch hemorrhage, among whom the position of the hemorrhage was in the branch of the hepatic artery $(n=9)$, superior mesenteric artery $(n=7)$, left gastric artery $(n=4)$, and dorsal pancreatic artery $(\mathrm{n}=2)$ (Table 2). As shown in Table 3, $81.8 \%$ (18/22) of cases underwent embolization 1 time. Intervention was applied twice in 4 participants due to rebleeding, and 1 of them was treated with a third intervention. Overall, $77.3 \%(17 / 22)$ of participants who underwent superselective embolization stopped hemorrhaging immediately after the procedure. Only 1 participant suffered reversible liver function impairment after embolization. No bowel or pancreatic necrosis was detected. A total of 5 participants who experienced rebleeding after embolization underwent a second traditional laparotomy. The second surgery stopped the bleeding in 3 of these participants, 2 participants failed to achieve hemostasis, and 1 died of pneumonia. The mortality rate was $13.6 \%(3 / 22)$.

A total of 53 participants had experienced arterial trunk hemorrhage. Among them, the position of the hemorrhage was in the stump of the gastroduodenal artery $(n=27)$, common hepatic artery $(n=12)$, proper hepatic artery $(n=6)$, right hepatic artery $(\mathrm{n}=5)$, aberrant right hepatic artery $(\mathrm{n}=2)$, and splenic artery $(\mathrm{n}=1)$. Covered stent placement succeeded in 32 cases $(60.4 \%)$, and the other participants (39.6\%) underwent embolization including distal and 
Table 4 Outcome of covered stent placement compared embolization in arterial trunk hemorrhage

\begin{tabular}{lccc}
\hline Outcome & $\begin{array}{c}\text { Embolization } \\
\text { group } \\
(\mathrm{n}=21)\end{array}$ & $\begin{array}{c}\text { Covered stent } \\
\text { placement group P value } \\
(\mathrm{n}=32)\end{array}$ & 0.132 \\
\hline Times of intervention & 18 & 31 & \\
Single & 3 & 1 & 0.283 \\
Multiple & 5 & 4 & \\
Hemostasis & 16 & 28 & 0.026 \\
No & & & \\
Yes & & 30 & \\
Complications of & 15 & 2 & 0.118 \\
intervention & 6 & & \\
No & & & \\
Yes & & & \\
Survival & 13 & & \\
No & & & \\
Yes & & & \\
\hline
\end{tabular}

proximal embolization (Table 2). As shown in Table 4, 1 participant in the stent group underwent a repeat stent placement due to the first stent not covering the artery leakage. A total of 3 participants in the embolization group underwent repeat embolization due to rebleeding. The multi-time intervention rates were $3.13 \%$ and $14.3 \%$ in the stent group and embolization group, respectively, but there was no significant difference between the 2 groups. The successful hemostasis rates were $87.5 \%$ and $76.2 \%$ in the stent group and embolization group, respectively, but there was no significant difference between the 2 groups. Complications of acute liver failure associated with the intervention were $6.25 \%$ and $28.6 \%$ in the stent group and embolization group, respectively, and chi-squared $\left(\chi^{2}\right)$ analysis showed a significant difference between the 2 groups $(\mathrm{P}=0.026)$. Complications of necrosis associated with intervention were not found. The mortality rates were $18.8 \%$ and $38.1 \%$ in the stent group and embolization group, respectively, but there was no significant difference.

A total of 10 patients experienced multisite hemorrhage. In this group, 8 participants had pancreatic or bile leakage accompanied with an abdominal infection. Embolization, covered stent placement, or a combination was applied, and only 2 participants stopped bleeding after the intervention procedure. A second surgery was performed on 3 participants, and only 1 case recovered. The total mortality rate was $70 \%$.

\section{Discussion}

Delayed PAH is a rare but life-threatening complication after hepatobiliary pancreatic surgery, and recent clinical studies have demonstrated that angiography with subsequent endovascular therapy would be the first-line approach for delayed PAH after hepatobiliary pancreatic surgery $(3,12)$. However, it is still unclear how to select the endovascular therapy method $(8,10)$. In this study, 85 delayed PAH patients who underwent endovascular therapy were enrolled, and cases were divided into 3 groups according to the arterial hemorrhage site. Then, our endovascular therapy choices for different sites of delayed PAH following hepatobiliary pancreatic surgery were concluded.

Former studies showed that Contrast-enhanced computed tomography (CT) prior to angiography proved to be helpful for detection of bleeding sites in patients with suspected hemorrhage $(1,2)$. But positive detection rate of contrast-enhanced CT was lower than selective arteriography (13). And contrast-enhanced CT may prolong the bleeding time, so emergency angiography should be performed immediately whenever sentinel bleeding is suspected. In our experience, contrast-enhanced CT may only recommend in hemodynamically stable patients.

In arterial branch hemorrhage, superselective embolization was recommended as the first choice (2). Several single-center studies previously demonstrated that superselective embolization is a technically and clinically effective procedure in patients with delayed postpancreatectomy hemorrhage (14). In our study, the successful hemostasis rate was $77.3 \%(17 / 22)$, which was similar to that of former studies. Only 1 participant experienced reversible liver function impairment after embolization, and no bowel or pancreatic necrosis was detected in our study. The complication rate associated with embolization was $4.5 \%$, which was lower than that in previous studies (14). The lower complication rate may be associated with our embolization strategy. When the hemorrhage site was the SMA branch or pancreatic artery, embolization was performed by coil only, with neither microspheres nor a gelatin sponge used for coil-assisted embolization. Several cases reported acute necrotizing pancreatitis after ectopic embolism during the transarterial 
chemoembolization (TACE) procedure (15-17), as a small particle embolizing agent, such as microspheres or a gelatin sponge, may give rise to ischemic pancreatitis. To prevent ischemic pancreatitis or bowel issues, we avoided using microspheres or a gelatin sponge in SMA branches or pancreatic arteries embolization. The results were encouraging, with no bowel or pancreatic necrosis detected in our study. The results suggested that superselective embolization with a coil only was a safe choice for hemorrhage from the SMA branches or pancreatic artery.

Endovascular stent-graft exclusion was first used in abdominal aortic aneurysm, and this endovascular method was widely used in arterial dissection and aneurysm (18). Some authors reported several cases in which covered stent placement was used to exclude the aneurysm or arterial leakage in delayed PAH after hepatobiliary pancreatic surgery $(8,11)$; however, there was no comparison between covered stent placement and embolization. In our study, there were 53 participants with arterial trunk hemorrhage, among whom 32 cases successfully underwent covered stent placement. The other 21 participants failed stent placement due to tortuous artery, thus, distal and proximal embolization were applied. The results showed that the covered stent placement group had a shorter intervention time, lower complication rate, and lower mortality than the embolization group. Moreover, the covered stent group had a more successful hemostasis rate than the embolization group. Although only the complication rate was significantly different due to the limitation of small sample size, covered stent placement showed its advantages compared to embolization. What's more, Distal and proximal embolization always needed several coils of different types or diameters. However, covered stent placement only needed single stent in most cases. Through, there was not excise comparison of operation time between the two methods, in our experience, covered stent placement need less time and easier to operate compared to embolization.

Covered stents can exclude artery leakage and protect the artery from further erosion caused by digestive fluid leakage or intra-abdominal infection, which was a key reason why the intervention time was low in the stent placement group $(4,19)$. The other reason was that the covered stent seldom migrated compared to embolization. In our population, it was observed in a rebleeding pseudoaneurysm case that the coil was rushed into a distal section of the vessel due to blood pressure recovery after first embolization (Figure 2). Thus, covered stent placement may decrease the rebleeding rate compared to embolization.
Covered stent can maintain the blood supply of the distal organ. Thus, the complication rate would be lower than with embolization (2). In our study, the complication rates associated with intervention were $6.25 \%$ and $28.6 \%$ in the stent group and embolization group, respectively. Chisquared $\left(\chi^{2}\right)$ analysis showed a significant difference between the 2 groups $(\mathrm{P}=0.026)$. The only case of liver failure documented in the stent group was caused by ischemia, and angiography after 2 stent placements verified that there was no blood in the stent, which was akin to the effect of embolization (Figure 3). Though stent placement had many advantages, the rate of stent placement in the main hemorrhage group was only $60.4 \%$. Vascular tortuosity and the limit of the covered stents were the main obstacles. Perhaps a high-compliance covered stent could improve the stent placement rate. Now, no recommendation regarding the type of covered stent has been reported in the literature. Recently, stent-assisted coil embolization was used in visceral aneurysm or pseudoaneurysm (20). This technique blends the use of a bare metal stent and coils. The bare metal stent is more soft than covered stent, so which is easy to be deployed across the lesion to serve as a scaffold, and a catheter is then "nosed" into the interstices of the uncovered stent (20). The safety of coil embolization has improved markedly because the coils are now "caged" behind the stent. Stent-assisted coil embolization can be used in certain anatomically challenging lesions.

Multisite hemorrhage is a lethal and tricky complication after hepatobiliary pancreatic surgery (11). Despite the use of embolization, covered stent placement, and second surgery, the mortality rate was still high. A retrospective cohort study reported that pancreatic duct diameter $<0.4 \mathrm{~cm}$, abdominal infection, clinically biliary or pancreatic fistula and delayed gastric emptying were independent risk factors associated with late PAH (21). In our group, 80\% of cases experienced a pancreatic or bile leak accompanied by abdominal infection. So, biliary or pancreatic fistula and abdominal infection maybe early method to predict delayed postoperative arterial hemorrhage, and preventing leaks and infection might be considered an effective way to prevent multisite hemorrhage occurrence. Recently, in our center, a new invagination pancreaticojejunostomy was applied to decrease the pancreatic fistula (22).

In conclusion, endovascular therapy is becoming the first choice for delayed PAH following hepatobiliary pancreatic surgery. This study concluded that discerning between different endovascular therapy choices for different sites of delayed postoperative arterial hemorrhage and stent 

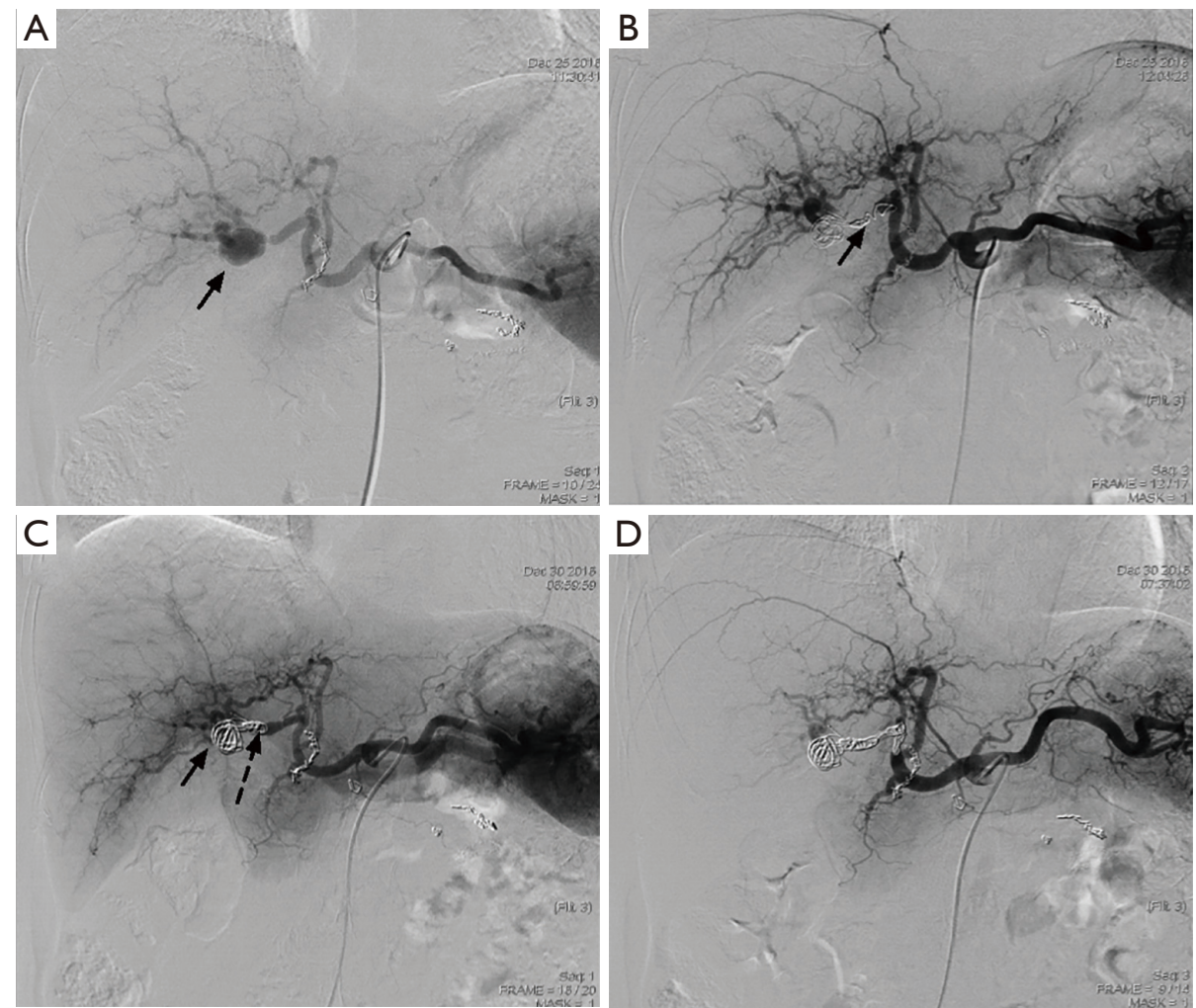

Figure 2 A rebleeding pseudoaneurysm located in branch of right hepatic artery that the coil was rushed into a distal section of the vessel due to blood pressure recovery after first embolization. (A) DSA showed delayed postoperative arterial hemorrhage caused by pseudoaneurysm located in branch of right hepatic artery after biliary tract surgery (arrow); (B) superselective embolization with coil (arrow) was applied and repeated DSA demonstrated a complete exclusion of the bleeding site; (C) five days after the first embolization, rebleeding was detected and DSA showed that the coil was rushed into the distal section of the vessel due to blood pressure recovery after first embolization (arrow); (D) a repeated superselective embolization with coil (arrow) was applied and repeated DSA demonstrated a complete exclusion of the bleeding site. DSA, digital subtraction angiography.
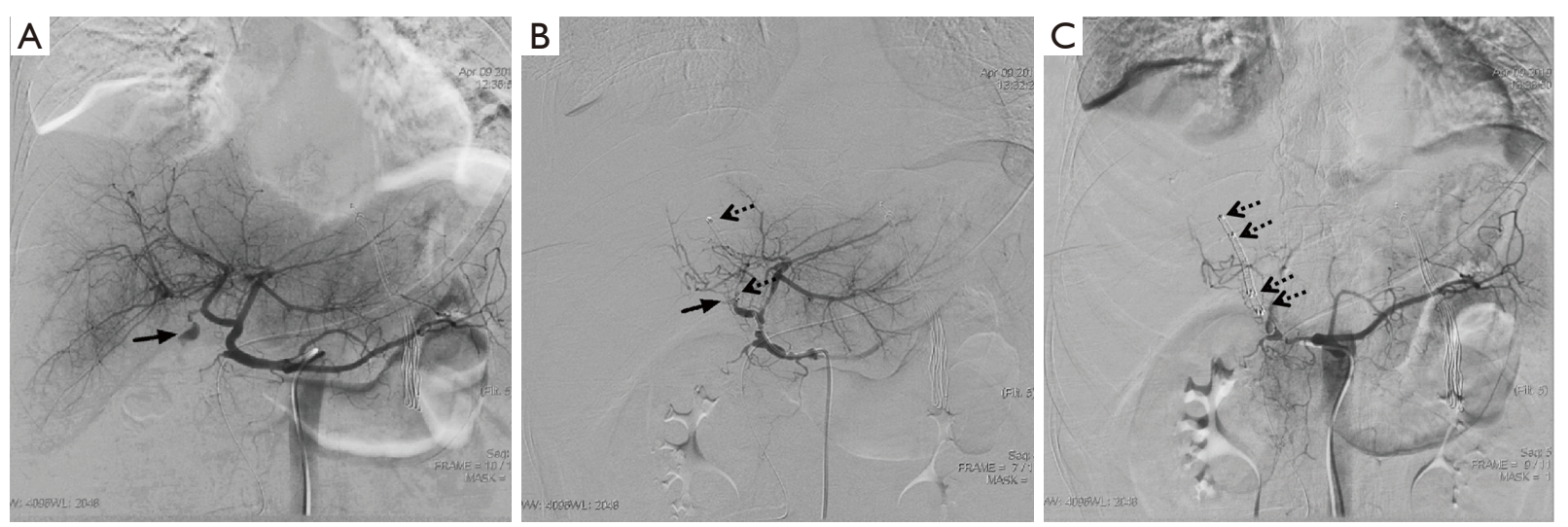

Figure 3 A right hepatic artery leakage after pancreatic surgery case treated by repeated cover stent placement suffered from liver failure due to ischemia. (A) DSA showed delayed postoperative arterial hemorrhage caused by leakage (solid arrow) located in right hepatic artery after pancreatic surgery; (B) first stent placement (dotted arrow) failed to cover the leakage (solid arrow) of the vessel; (C) after the second stent placement (dotted arrow), there was no arterial blood supply to liver, and the patient experienced liver failure caused by ischemia. DSA, digital subtraction angiography. 
placement may provide the optimal first choices for delayed PAH with localization of the arterial trunk. Limited by the sample size, the conclusion needs to be verified by additional large population studies.

\section{Acknowledgments}

Funding: This study was supported by the National Natural Science Foundation of China (No. 31971249).

\section{Footnote}

Reporting Checklist: The authors have completed the STROBE reporting checklist. Available at https://dx.doi. org/10.21037/gs-21-521

Data Sharing Statement: Available at https://dx.doi. org/10.21037/gs-21-521

Conflicts of Interest: All authors have completed the ICMJE uniform disclosure form (available at https://dx.doi. org/10.21037/gs-21-521). The authors have no conflicts of interest to declare.

Ethical Statement: The authors are accountable for all aspects of the work in ensuring that questions related to the accuracy or integrity of any part of the work are appropriately investigated and resolved. All procedures performed in this study involving human participants were in accordance with the Declaration of Helsinki (as revised in 2013). The study was approved by ethics board of Eastern Hepatobiliary Surgery Hospital, and informed consent was taken from all the patients.

Open Access Statement: This is an Open Access article distributed in accordance with the Creative Commons Attribution-NonCommercial-NoDerivs 4.0 International License (CC BY-NC-ND 4.0), which permits the noncommercial replication and distribution of the article with the strict proviso that no changes or edits are made and the original work is properly cited (including links to both the formal publication through the relevant DOI and the license). See: https://creativecommons.org/licenses/by-nc-nd/4.0/.

\section{References}

1. Chen JF, Xu SF, Zhao W, et al. Diagnostic and therapeutic strategies to manage post-pancreaticoduodenectomy hemorrhage. World J Surg 2015;39:509-15.

2. Chang YC, Liu KL, Huang YC, et al. Efficacy and hepatic complications of three endovascular treatment approaches for delayed postpancreatectomy hemorrhage: evolution over 15 years. CVIR Endovasc 2019;2:33.

3. Feng J, Chen YL, Dong JH, et al. Postpancreaticoduodenectomy hemorrhage: risk factors, managements and outcomes. Hepatobiliary Pancreat Dis Int 2014;13:513-22.

4. Liang X, Shi LG, Hao J, et al. Risk factors and managements of hemorrhage associated with pancreatic fistula after pancreaticoduodenectomy. Hepatobiliary Pancreat Dis Int 2017;16:537-44.

5. Klaiber U, Mihaljevic A, Hackert T. Radical pancreatic cancer surgery-with arterial resection. Transl Gastroenterol Hepatol 2019;4:8.

6. Dumitru R, Carbunaru A, Grasu M, et al. Pseudoaneurysm of the splenic artery - an uncommon cause of delayed hemorrhage after pancreaticoduodenectomy. Ann Hepatobiliary Pancreat Surg 2016;20:204-10.

7. Mimatsu K, Fukino N, Kano H, et al. Surgical Laparotomy for Repeated Delayed Arterial Hemorrhage after Pancreaticoduodenectomy. Case Rep Gastroenterol 2019;13:50-7.

8. Zhou TY, Sun JH, Zhang YL, et al. Postpancreaticoduodenectomy hemorrhage: DSA diagnosis and endovascular treatment. Oncotarget 2017;8:73684-92.

9. Huo Y, Chi J, Zhang J, et al. Endovascular intervention for delayed post-pancreaticoduodenectomy hemorrhage: clinical features and outcomes of transcatheter arterial embolization and covered stent placement. Int J Clin Exp Med 2015;8:7457-66.

10. Hassold N, Wolfschmidt F, Dierks A, et al. Effectiveness and outcome of endovascular therapy for late-onset postpancreatectomy hemorrhage using covered stents and embolization. J Vasc Surg 2016;64:1373-83.

11. Ching KC, Santos E, McCluskey KM, et al. Covered Stents and Coil Embolization for Treatment of Postpancreatectomy Arterial Hemorrhage. J Vasc Interv Radiol 2016;27:73-9.

12. Khalsa BS, Imagawa DK, Chen JI, et al. Evolution in the Treatment of Delayed Postpancreatectomy Hemorrhage: Surgery to Interventional Radiology. Pancreas 2015;44:953-8.

13. Muglia R, Lanza E, Poretti D, et al. Emergency endovascular treatments for delayed hemorrhage after pancreaticobiliary surgery: indications, outcomes, and follow-up of a retrospective cohort. Abdom Radiol (NY) 
2020;45:2593-602.

14. Stampfl U, Hackert T, Sommer CM, et al. Superselective embolization for the management of postpancreatectomy hemorrhage: a single-center experience in 25 patients. $\mathrm{J}$ Vasc Interv Radiol 2012;23:504-10.

15. Bae SI, Yeon JE, Lee JM, et al. A case of necrotizing pancreatitis subsequent to transcatheter arterial chemoembolization in a patient with hepatocellular carcinoma. Clin Mol Hepatol 2012;18:321-5.

16. Yamaguchi T, Seki T, Komemushi A, et al. Acute necrotizing pancreatitis as a fatal complication following DC Bead transcatheter arterial chemoembolization for hepatocellular carcinoma: A case report and review of the literature. Mol Clin Oncol 2018;9:403-7.

17. Tan Y, Sheng J, Tan H, et al. Pancreas lipiodol embolism induced acute necrotizing pancreatitis following transcatheter arterial chemoembolization for hepatocellular carcinoma: A case report and literature review. Medicine (Baltimore) 2019;98:e18095.

Cite this article as: Yu X, Liu X, Huang J, Shen S, Ge N, Yang Y, Wang H. Endovascular therapy choices for different sites of delayed postoperative arterial hemorrhage after hepatobiliary pancreatic surgery: a retrospective study. Gland Surg 2021;10(9):2745-2753. doi: 10.21037/gs-21-521
18. de Beaufort H, Zandvoort HJ, Moll FL, et al. The Endurant stent graft for endovascular aneurysm repair. Expert Rev Med Devices 2014;11:331-40.

19. Frego M, Bertin M, Brolese A, et al. Endovascular treatment of acute pseudoaneurysm associated to pancreaticoduodenectomy. Hepatogastroenterology 2009;56:1738-41.

20. Jesinger RA, Thoreson AA, Lamba R. Abdominal and pelvic aneurysms and pseudoaneurysms: imaging review with clinical, radiologic, and treatment correlation. Radiographics 2013;33:E71-96.

21. Lu JW, Ding HF, Wu XN, et al. Intraabdominal hemorrhage following 739 consecutive pancreaticoduodenectomy: Risk factors and treatments. J Gastroenterol Hepatol 2019;34:1100-7.

22. Li B, Xu C, Qiu ZQ, et al. An end-to-side suspender pancreaticojejunostomy: A new invagination pancreaticojejunostomy. Hepatobiliary Pancreat Dis Int 2018;17:163-8. 